\title{
Classical T Tauri stars: accretion, wind, dust
}

\author{
P.P. Petrov
}

Crimean Astrophysical Observatory, Nauchny, Crimea, 298409

e-mail: petrov@craocrimea.ru

Submitted on September 23, 2020

\begin{abstract}
Classical T Tauri stars (CTTS) are at the early evolutionary stage when the processes of planet formation take place in the surrounding accretion disks. Most of the observed activity in CTTS is due to magnetospheric accretion and wind flows. Observations of the accreting gas flows and appearance of the line-dependent veiling of the photospheric spectrum in CTTS are considered. Evidence for the dusty wind causing the observed irregular variability of CTTS is presented. Photometric and spectroscopic monitoring of two CTTS, RY Tau and SU Aur, has been carried out at the Crimean Astrophysical Observatory since 2013 aimed at studying the dynamics of accretion and wind flows on time scales from days to years. The observed variations in the dynamical parameters may be caused by changes in the accretion rate and in the global magnetic fields of CTTS.
\end{abstract}

Key words: young stars, RY Tau, SU Aur, RW Aur

\section{Introduction}

T Tauri stars were first investigated and distinguished into a new type of variables 75 years ago (Joy, 1945). Among distinctive features there is an irregular brightness variability, an emission spectrum of low excitation, and association with dark gas and dust clouds. V.A. Ambartsumyan was the first to conclude about evolutionary youth of these stars (Ambartsumyan, 1949).

The classical T Tauri stars (CTTS) are currently referred to as young low-mass stars $\left(\leq 2 \mathrm{M}_{\odot}\right)$ with accretion disks. The life time of the accretion disk on average accounts for about ten million years. After the formation of the planetary system and dissipation of the accretion disk stars become less active and proceed to the class of the so-called "Weak-line TTS" (WTTS). Basic information on the T Tauri stars can be found in the reviews of Petrov (2003), Bouvier et al. (2007), Hartmann et al. (2016).

Contrary to the more massive $\left(>2 \mathrm{M}_{\odot}\right.$ ) young stars related to the class of Herbig Ae/Be stars (HAeBe), CTTS have convective envelopes and, consequently, strong magnetic fields and pronounced "magnetic-type" activity, in particular, X-ray emission (Pevtsov et al., 2003). This is a significant difference since the interaction of the accretion disk with the magnetic field generates most of the observed manifestations of CTTS activity.

Modern ideas about the physical processes that occur on T Tauri stars are based on the magnetospheric accretion model. This model was developed for neutron stars (Ghosh and Lamb, 1979) and later applied to T Tauri stars (Camenzind, 1990; Koenigl, 1991). Contrary to the compact objects where the fall time of matter is measured in milliseconds (neutron stars) or minutes (white dwarfs incorporated in cataclysmic variables), this time is about a day in CTTS. This allows one to observe motions of gas flows with sufficient time resolution and carry out a detailed comparison of the observed phenomena with what yields the numerical modeling of magnetohydrodynamic (MHD) processes.

Table 1. Spectral types of CTTS

\begin{tabular}{lll}
\hline Star & Spectrum & Reference \\
\hline SU Aur & G2 III & Herbig and Bell (1988) \\
V1331 Cyg & G7-K0 IV & Petrov et al. (2014b) \\
RY Tau & K1 IV & Herbig and Bell (1988) \\
RW Aur A & K1 & Herbig and Bell (1988) \\
RW Aur B & K5 IV & Dodin et al. (2020) \\
S CrA SE & K6 IV & Gahm et al. (2018) \\
DR Tau & K7 V & Petrov et al. (2011) \\
\hline
\end{tabular}

The paper provides a brief review of the results of observing accretion and wind of the selected CTTS derived with the author's participation in different years and the new results of studies carried out at the Crimean Astrophysical Observatory. The spectral types of stars discussed in the paper are given in Table 1.

\section{Accretion and veiling of the photospheric spectrum}

Observations with the ALMA interferometer in the millimeter wavelength range first provided an opportunity to derive 
images of the circumstellar disks of a number of CTTS, determine their sizes, masses, inclination angles and recognize signatures of planet formation in the structure of these disks (Dong et al., 2018; Long et al., 2019). Most inner regions of accretion disks were observed earlier with interferometers in the near IR range. Sizes of the inner boundary of the dusty disk of CTTS are 0.05-0.2 a.u. (Akeson et al., 2005). Magnetospheres of CTTS are of less sizes and are spatially unresolved yet. Nonetheless, it is possible to determine the direction and radial velocities of gas flows inside the magnetosphere and in the wind using the spectral line profiles.

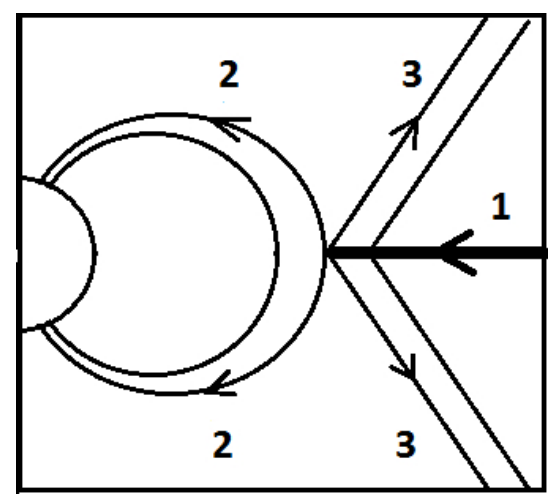

Fig. 1. Basic directions of accretion and wind flows: $1-$ disk accretion, 2 - magnetospheric accretion, 3 - wind

Figure 1 schematically shows the directions of the accretion and wind flows in the magnetospheric accretion model. At a quite large inclination angle of the magnetic dipole axis the line-of-sight intersects both the wind region and the magnetospheric accretion region. Figure 2 exhibits the hydrogen Balmer line profiles in the spectrum of S CrA SE (Gahm et al., 2018).
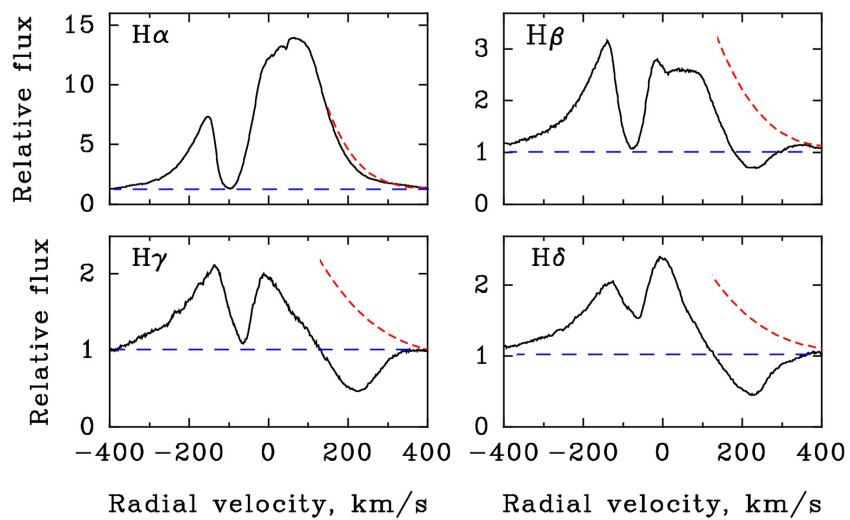

Fig. 2. Balmer lines in the spectrum of S CrA SE. In the long-wavelength wing, the dashed lines mark the short-wavelength wing region that is symmetrically "reflected" with respect to the zero velocity. The wavelength scale is astrocentric

The typical profile of the $\mathrm{H}_{\alpha}$ emission line in the spectrum of CTTS has a depression in the "blue" wing caused by absorption in the wind. This is a profile of the type B
II-III according to the classification of Reipurt et al. (1996). Absorption (below the continuum) in the infalling gas flow is well seen in high members of the Balmer series where emission is not so intensive. As good indicators of accretion we can note hydrogen lines in the IR range: $\mathrm{Pa} \beta, \mathrm{Br} \gamma$ (Folha and Emerson, 2001), and the He I $10800 \AA$ line. In the visible spectrum region, the inverse $\mathrm{P}$ Cyg profile is usually seen in the He I $5886 \AA$ line, in the Na I D resonance doublet lines, and in the O I $7773 \AA$ triplet lines.

In the spectra with a high signal-to-noise ratio one can see the signatures of accretion in the red-shifted absorption components of metal photospheric lines. Figure 3 shows the metal line profiles in the spectrum of S CRA SE. The highest infalling velocity is shown by the lines with high excitation potential $(8 \mathrm{eV})$ formed at the base of the accretion column, near the shock wave where the temperature and density of the infalling gas are the highest (Petrov et al., 2014a).

The other consequence of magnetospheric accretion is the veiling of the photospheric spectrum of CTTS. Hard radiation of the shock wave at the base of the accretion channel ionizes the infalling gas and also heats the stellar photosphere up to the optical thickness $\tau>1$ in the continuum. This results in the formation of a hot photospheric spot at a temperature of about $8000 \mathrm{~K}$ (Lamzin et al., 1996; Gullbring et al., 2000).

On the background of photospheric radiation of the late-type star such a spot yields the additional continuum which reduces contrast of the photospheric spectrum of the rest, undisturbed part of the photosphere. In the observed stellar spectrum, the equivalent widths EW of photospheric lines appear to be reduced. As a quantitative expression for this effect there serves the so-called veiling factor $\mathrm{VF}=\mathrm{EW}(\mathrm{sp}) / \mathrm{EW}(\mathrm{obs})-1$, where $\mathrm{EW}(\mathrm{sp})$ is the equivalent width of the photospheric line, which corresponds to the spectral type of the undisturbed photosphere, whereas $\mathrm{EW}$ (obs) is the observed equivalent width.

The observed spectra of CTTS show the veiling factors from zero to unity and more. However, a detailed study of this effect reveals a disagreement with the simple hot spot model. First of all, the model implies a correlation between the veiling and stellar brightness. If the veiling factor at the wavelength $5500 \AA$ is equal to unity, then the stellar brightness in the V band should be increased by $0.75^{m}$. In 1995-1999, at the NOT observatory there were carried out three series of simultaneous spectral and photometric observations of RW Aur A (Petrov et al., 2001). The stellar brightness and veiling factor varied in large ranges, however there were no correlation between them. This correlation was possibly cancelled out by variable circumstellar extinction.

A study of the veiling effect requires high spectral resolution and high signal-to-noise ratio in order to measure equivalent widths of the weakest lines. Such measurements were performed in spectra of several CTTS obtained by G. Herbig at the Keck-1 telescope (Rei et al., 2018). Figure 4 shows the results of measuring the veiling of a number of spectral lines including the weakest ones with an equivalent width of $10 \mathrm{~m} \AA$. As a standard of the appropriate spectral type there were used the synthetic spectra calculated with the SME software package (Valenti and Piskunov, 1996). In Fig. 4, the ratio of equivalent widths EW(synth)/EW(obs) that is equal to unity corresponds to $\mathrm{VF}=0$. One can see that the majority of lines show a significant veiling level, whereas 

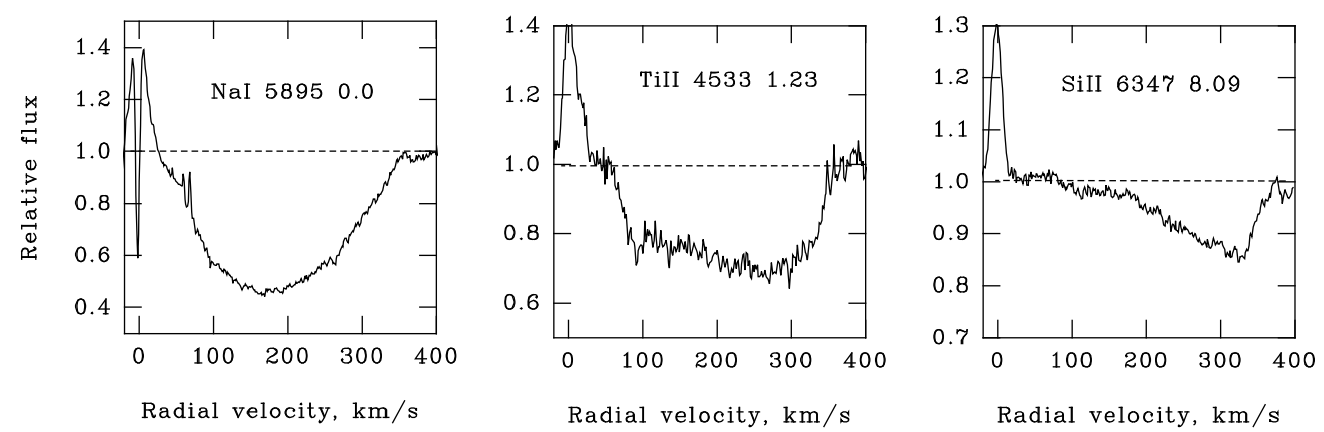

Fig. 3. Profiles of the lines formed in the accretion flow of S CrA SE
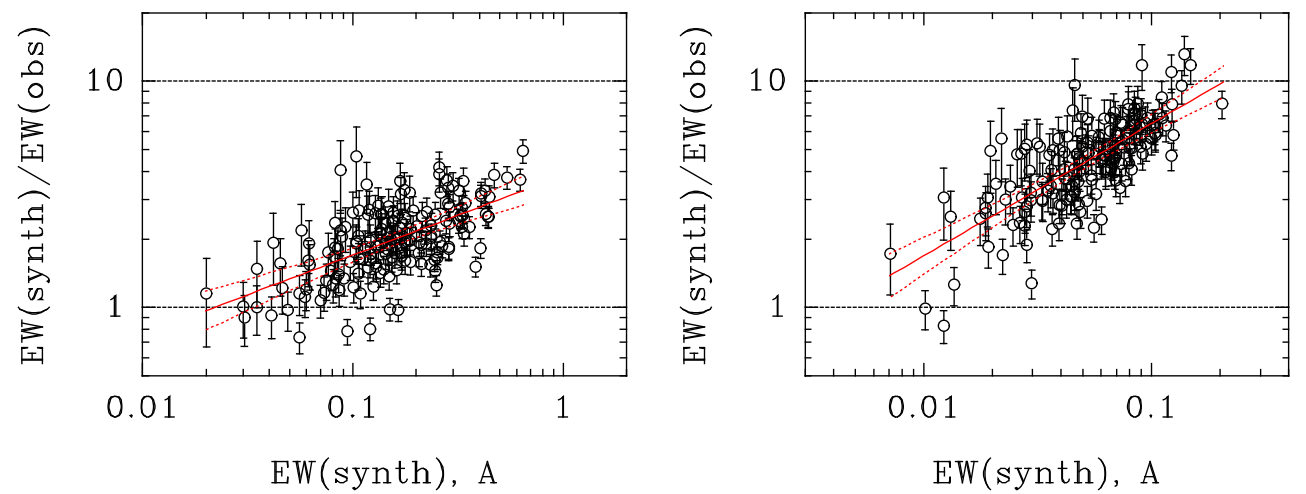

Fig. 4. Ratio of equivalent widths of photospheric lines in the spectrum of the standard and CTTS: LkHa 321 Cyg to the left, V1331 to the right

the weakest ones are not changed, i.e., a hot photospheric spot on the surfaces of these stars is evidently absent.

In a series of spectra of DR Tau derived with the NOT telescope in 2007, the equivalent widths of a great number of photospheric lines of different intensity were measured. As well as in the case above, the strong lines (of more oscillator's force) turned out to be more affected by veiling than the weak lines. During several nights the veiling level changed, but the indicated effect persisted (Petrov et al., 2011).

Within the magnetospheric accretion model this means that accretion changes the temperature profile of the photosphere: upper layers are heated by the shock wave radiation, whereas the lower ones remain unchanged. There arises a "chromospheric spot". In the observed stellar spectrum, the photospheric absorptions are filled with chromospheric emission, and this causes the veiling effect. It is worth noting that a similar explanation of veiling was mentioned in the first paper of Joy (1945) where the spectral features of T Tauri stars were described.

The region of enhanced chromospheric emission caused by accretion distorts the observed photospheric line profiles. If the "chromospheric spot" is shifted with respect to the stellar rotation pole, then the radial velocities measured from photospheric absorptions and narrow emission lines, for instance He I $5876 \AA$, change in opposite phase. Such an effect was observed for several CTTS: DR Tau, DI Cep, RU Lup, RW Aur A, S CrA SE (Petrov et al., 2011).

When the accretion flow is enhanced, the deeper layers of the photosphere are heated and the "hot spot" is formed that radiates in the continuum. The process of heating of the CTTS photosphere by radiation of the shock wave and the formation of emission in both the continuum and lines were considered in the paper of Dodin, Lamzin (2012).

Although hot spots produced by accretion have effect on the apparent stellar brightness, the observed irregular brightness variability of CTTS is often due to circumstellar extinction. This is well seen on the example of the RY Tau star since it is observed at a large inclination angle and the line-of-sight intersects the dusty disk atmosphere. During variations of stellar brightness within one magnitude the veiling in the visible spectrum region does not vary (Babina et al., 2016). This means that the reason for brightness variation is circumstellar extinction. Observations indicate that the dust screen is near the star and is apparently a dusty wind near the radius of dust sublimation (see the next section).

\section{Wind and circumstellar dust}

The outflow (wind) occurs beyond the limits of the CTTS magnetosphere where gas flows along the open lines of force of the large-scale magnetic field. Though the term "wind" is used, the mechanisms of a CTTS outflow are different from those that are responsible for the solar wind or the wind of hot stars. Several types of the wind of young stars were suggested and considered in detail in the literature.

The disk wind starts from the extended surface of the accretion disk and is accelerated by the magnetic centrifuge: the 
matter flows along open lines of force of the disk magnetic field (Matt and Pudritz, 2005). The $X$-wind starts from the boundary of the magnetosphere and disk and is accelerated by the magnetic centrifuge (Shu et al., 1994). Also at the inner disk, a conical wind can arise, accelerated by the magnetic pressure of the azimuthal component of the magnetic field at the boundary of the magnetosphere (Romanova et al., 2009). The propeller mechanism arises in the case when the angular velocity of the star and magnetosphere exceeds the angular velocity of the Keplerian rotation on the inner disk boundary (Romanova et al., 2018). This may occur with decreasing rate of disk accretion or enhancing stellar magnetic field. Otherwise, when the inner disk rotates faster than the magnetosphere, there arise the bipolar magnetic ejections of matter due to inflation of the magnetosphere and reconnection of lines of force - the magnetosphere constantly "rearranges" itself (Goodson et al., 1997; Zanni and Ferreira, 2013). In the polar region of CTTS, there can also arise an outflow along open lines of the stellar magnetic field, the so-called polar wind (Cranmer, 2008).

From the listed above outflow mechanisms one can see that the main reason of outflowing is accretion - disk or magnetospheric. The magnetic field (of a disk or a star) redirects a part of the accreting matter into the outflow. In this way, the system "star + disk" releases the excess angular momentum.

One cannot always establish from observations which outflow mechanism is in operation in each specific case. Observers analyze spectral line profiles broadened due to the Doppler effect and their variability in time. A discussion concerning emission lines in CTTS spectra within accretion and wind models can be found in Hartmann (1998). The atlas of the observed $\mathrm{H}_{\alpha}$ emission profiles in spectra of CTTS and $\mathrm{HAeBe}$ is represented in Reipurt et al. (1996).

Analyzing CTTS spectra, Alencar and Basri (2000) found that broad emission lines are formed both inside the magnetosphere in the flows of infalling gas and outside it, in the wind flows. The model calculations performed by Kwan and Fischer (2011) showed that the broad emission lines of $\mathrm{H}_{\mathrm{I}}$ and He $\mathrm{r}$ in CTTS spectra are formed in the radial wind flows at the electron density $\mathrm{N}_{\mathrm{e}}=10^{9} \mathrm{~cm}^{-1}$ and temperature $\mathrm{T}_{\mathrm{e}} \sim 10^{4} \mathrm{~K}$, whereas the $\mathrm{Ca}$ II lines - in magnetospheric flows and on the inner disk boundary at $\mathrm{N}_{\mathrm{e}}=10^{12} \mathrm{~cm}^{-1}$ and $\mathrm{T}_{\mathrm{e}} \leq 7500 \mathrm{~K}$.

Analyzing RW Aur A spectra, Alencar et al. (2005) came to the conclusion that the Balmer lines are generally formed in the narrowly collimated wind which starts on the inner disk boundary. With more extended region of the disk wind the observed emission line profiles are not reproduced well.

Models of the disk wind and magnetospheric accretion were used to calculate spectral line profiles (see, e.g., Kurosawa et al., 2011; Dmitriev et al., 2019). A comparison of the calculated profiles with the observed ones allows us to ascertain parameters of models and estimate the mass loss rate. One of the parameters: V1331 Cyg - a CTTS that is visible from the pole side. A comparison of the observed Balmer line profiles with the calculated ones within the magnetosphere and disk wind models made it possible to determine the mass loss rate. Variability of the P Cyg-type profile on a time scale of a few years was shown to be caused by variations of the disk wind collimation angle within a few degrees and the mass loss rate within $(6-11) \cdot 10^{-8} \mathrm{M}_{\odot} / \mathrm{yr}$ (Petrov et al., 2014a).
Another example: the binary star S CrA that consists of two CTTS - S CrA SE and S CrA NW. The modeling of Balmer line profiles made it possible to determine that the inclination angle of the rotation axis of each star accounts for 65 degrees (Gahm et al., 2018), whereas the interferometric observations in the near IR range indicated a small inclination angle of the accretion disk. This divergence is probably caused by the fact that in the observed IR radiation of the disk there is also thermal dust radiation from the disk wind (Bans and Koenigl, 2012), which hampers the determination of the disk inclination angle.

Interferometry of CTTS in the near IR range shows that the inner boundary of the dust disk (dust sublimation zone) is at a distance of 0.05-0.2 a.u. (Akeson et al., 2005). This is already close to the zone of outflow formation (magnetospheric ejections, propeller wind, etc.). Tambovtseva and Grinin (2008) showed that dust can exist in the wind of CTTS, and the dusty wind is a reason for the circumstellar extinction.

The spectral and photometric monitoring of CTTS RY Tau throughout a few years made it possible to detect that during the episodes of intensive outflowing the circumstellar extinction decreased, which was interpreted as an influence of magnetospheric ejections on the dusty disk wind (Babina et al., 2016). The physical mechanism of this phenomenon is not clear. One can suggest that the hot gas ejected during the magnetosphere's opening "sweeps off" the dusty disk wind on the inner boundary of the dust disk. In the intervals between such ejections the dust wind raises dust to the lineof-sight, and the circumstellar extinction is restored. It is also worth noting that the magnetospheric ejection should have effect on the disk magnetic field, which can contribute disturbances into the wind flow on the inner disk boundary. In any case the found dependence between gas ejection and variation in circumstellar extinction allows one to ascertain the distance at which the dust screen is located. The time lag between these two events does not exceed a day. At an ejection velocity of about $200 \mathrm{~km} \cdot \mathrm{s}^{-1}$ gas travels a distance of $\sim 0.1$ a.u. per day, which coincides with the distance of dust sublimation (inner boundary of the dust disk). Thus, one can suggest that in the case of RY Tau the dust screen is hot dust in the disk wind at the distance of sublimation, as it is schematically shown in Fig. 5.

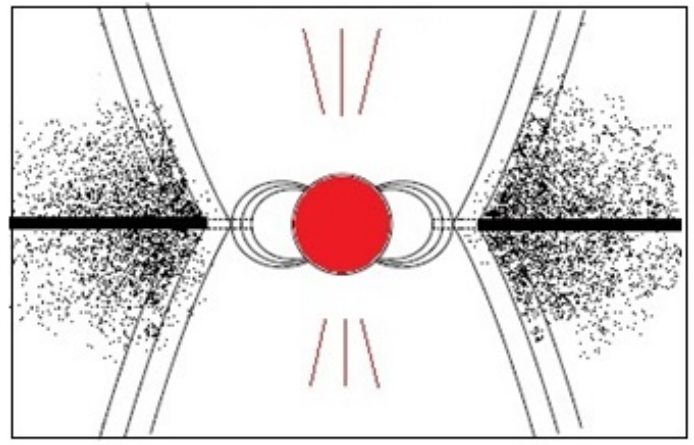

Fig. 5. Dusty disk wind of RY Tau

The conclusion that RY Tau is seen through the dusty atmosphere of the inner accretion disk has also been obtained 
when analyzing interferometric observations of RY Tau in the visible and near IR ranges (Davies et al., 2020). The similar result was acquired for SU Aur: the hot dust above the disk plane is a necessary ingredient for interpreting the excess of radiation at a wavelength of $2.2 \mathrm{mkm}$ (Labdon et al., 2019).

The other example showing the presence of hot dust in the disk wind is the binary system RW Aur. The major component, RW Aur A, is one of the brightest and most frequently observed CTTS. The secondary, less massive component, RW Aur B, is on average three magnitudes weaker. The components are at an angular distance of $\approx 1.5 \mathrm{arcsec}$, which allows one to study them separately at a good image quality (Antipin et al., 2015). In the recent paper of Dodin et al. (2020), RW Aur was shown to exhibit characteristics of the UX Ori-type star. Since 2010 the significant decreases of the apparent brightness of RW Aur A were observed down to three stellar magnitudes on a time scale of about a year, thus the major component was sometimes weaker than the secondary one. Such deep and prolonged decreases in brightness have occurred in other CTTS and were interpreted as eclipses by dust clouds. In the case of RW Aur A, the hypothesis was first suggested that absorbing dust is at a significant distance from the star, in the tidal gas and dust arm which is formed when the secondary component passes through the periastron (Rodriguez et al., 2018). However, photometric observations showed that during a decrease of the radiation flux from RW Aur A in the visible wavelength, the flux at wavelengths $2-5 \mu \mathrm{m}$ increases simultaneously (Shenavrin et al., 2015). In this wavelength range, dust radiates near the temperature of sublimation. The authors assumed that decreases in brightness in the visible range were caused by the appearance of hot dust in the disk wind of RW Aur A at a temperature of about $1000 \mathrm{~K}$. A similar interpretation was previously proposed by Grinin et al. (2009) to explain the deep minimum of the T Tauri star V1184 Tau, where the decrease in brightness in the optical range with constant or slightly increased emission in the $\mathrm{K}$ band was caused by an increase in the disk thickness near the dust evaporation zone as a result of amplification of the disk wind.

Dodin et al. (2019) recorded a significant increase in the linear polarization of RW Aur A during deep minima in 2010-2011 and 2014-2018. The value and orientation of the polarization vector indicated that the star and inner regions of the accretion disk were shielded by the axial symmetric dusty disk wind. The spectral observations of Takami et al. (2016) detected that during the brightness minimum in the spectrum of RW Aur almost all the photospheric lines disappeared, except for the strongest ones. Such a phenomenon was first predicted and modeled by Grinin et al. (2006). During the deep brightness minimum the star is covered by dust and the scattered light is generally observed. While scattering stellar radiation by moving dust particles in the inner regions of the circumstellar disk and disk wind, the spectral lines become broader due to the Doppler effect. As a result, weak lines become blurring, practically invisible.

An order of magnitude increase of the iron abundance in the corona of RW Aur A was recorded by the Chandra X-ray Observatory during the brightness minimum in 2017. It was assumed that the destruction of planetesimals or an Earth-type planet could lead to a significant increase of dust which was accreted onto the star (Günther et al., 2018).

\section{Dynamics of accretion and wind flows}

Broad emission line profiles in the spectrum of CTTS provide a notion on the direction and velocity of gas flows around the star. The review and classification of emission line profiles in the spectra of CTTS are given in Reipurt et al. (1996). Analysis of emission line profiles of hydrogen and metals in the spectra of 30 CTTS was carried out by Alencar and Basri (2000). The formation of various types of the $\mathrm{H}_{\alpha}$ emission profile in the spectra of CTTS was considered within the magnetosphere and disk wind models (Kurosawa et al., 2006).

The processes of accretion and outflows are substantially nonstationary. Observations of variability of spectral line profiles can yield additional information on the medium where these lines are formed. The extended series of spectral observations were carried out only for several CTTS (see Table 2).

Table 2. Series of spectral observations of CTTS

\begin{tabular}{lcl}
\hline Star & Observational period & Reference \\
\hline SU Aur & $1986-1990$ & Giampapa et al. (1993) \\
& $1986-1992$ & Johns and Basri (1995) \\
& $1990-1994$ & Petrov et al. (1996) \\
& $2013-2019$ & Petrov et al. (2019) \\
RW Aur A & $1995-1999$ & Petrov et al. (1996) \\
& $1989-1999$ & Alencar et al. (2005) \\
& $2010-2015$ & Takami et al. (2016) \\
DR Tau & $1989-1998$ & Alencar et al. (2001) \\
RY Tau & $2013-2019$ & Babina et al. (2016), \\
& & Petrov et al. (2019) \\
\hline
\end{tabular}

The SU Aur (G2 III) star with a mass of $2 \mathrm{M}_{\odot}$ occupies an intermediate position between CTTS and HAeBe. RW Aur $\mathrm{A}$ is a typical CTTS and is distinguished by a high accretion rate (White and Ghez, 2001). Both stars are oriented in such a way that the line-of-sight intersects both the wind region and accretion region. Meanwhile, in addition to nonstationary processes of accretion and wind, it is possible to detect periodic variations associated with the axial rotation of the star or the disk. In the spectrum of SU Aur, a period of about three days was detected in intensity variations of the "blue" wing of $\mathrm{H}_{\alpha}$ emission, as well as in variability of the $\mathrm{H}_{\beta}$ absorption component shifted to the red side. This was interpreted as a rotational modulation within the inclined magnetic dipole model (Giampapa et al., 1993; Johns and Basri, 1995). A similar effect of axial accretion asymmetry was also observed in RW Aur: in variations of radial velocity of broad emission lines there was detected a period of 2.77 days. Presumably, over one revolution of the star with a period of $\sim 5.5$ days, two poles of accretion pass the line-of-sight (Petrov et al., 2001). A period of 5.6 days was detected in magnetic field variations of RW Aur A (Dodin et al., 2012).

The prolonged series of spectral observations of the other CTTS, DR Tau (K7), includes 103 spectra obtained in 19891998 (Alencar et al., 2001). The star exhibits significant variations in emission line profiles on different time scales, whereas no period is observed. This seems to be associated with the disk orientation: images of the accretion disks of CTTS derived with the ALMA interferometer shows that DR 
Tau is seen at a small inclination angle ("pole-on") (Long et al., 2019). Since only one accretion pole is constantly seen, the rotational modulation is not revealed.
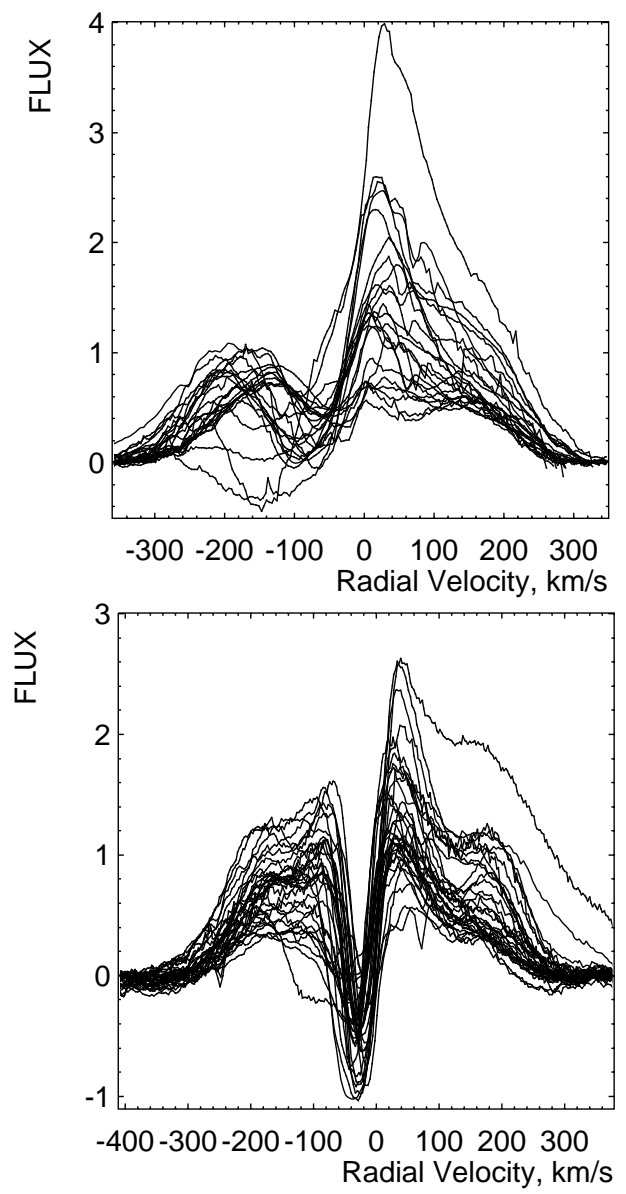

Fig. 6. Range of variations of the $\mathrm{H}_{\alpha}$ line in the spectrum of RY Tau (2014-2015) and in the spectrum of SU Aur (2017-2018). The flow is expressed in units of $3.67 \cdot 10^{13} \mathrm{erg} \mathrm{cm}^{-2} \mathrm{~s}^{-1} \AA^{-1}$
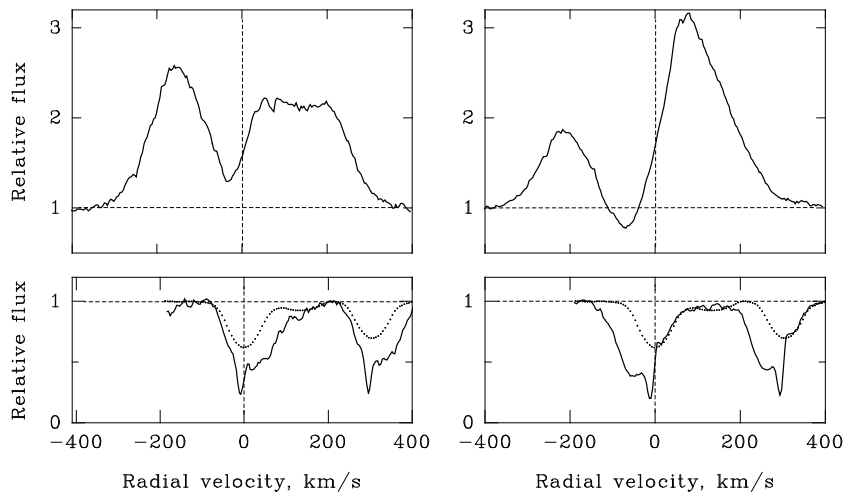

Fig. 7. $\mathrm{H}_{\alpha}$ and $\mathrm{Na}$ I D lines in the spectrum of RY Tau (HJD 2458737 and 2458765). In the Na I D lines, the dashed line marks the photospheric profile of the G2 III star broadened by stellar rotation, $\mathrm{v} \sin i=50 \mathrm{~km} / \mathrm{s}$. The scale of radial velocities is astrocentric

The spectral monitoring of two CTTS, RY Tau and SU Aur, has been carried out for several years at CrAO RAS.
Our spectral observations are accompanied by simultaneous photometry in the BVRI system. This makes it possible to determine radiation fluxes in emission lines in absolute units, which can be useful when interpreting the obtained data. The results of this monitoring over the first five seasons (20132018) are published in Petrov et al. (2019). As an example, Fig. 6 shows variations of the flow profile in the $\mathrm{H}_{\alpha}$ line during one season when these variations were maximal. The range of variations of the $\mathrm{H}_{\alpha}$ line changes noticeably from season to season for both stars.

In the absorption line profile of the $\mathrm{Na}$ I $\mathrm{D}$ resonance doublet in the spectrum of RY Tau one can clearly see the absorptions from the red and blue side from the photospheric line, arising in the incident and in the outflowing gas streams (see Fig. 7). Variations of these absorptions correlate with variations in the $\mathrm{H}_{\alpha}$ emission line profile.

The characteristic time of variations of $\mathrm{Na}$ I $\mathrm{D}$ absorptions formed in the infalling and outflowing gas is about 20 days. Moreover, there has been observed a variation in the mean intensity of these absorptions for a few years (Fig. 8). This possibly reflects a variation of the relative role of accretion and outflow.

The other star, SU Aur, also shows significant variations in the dispersion of the radiation flow in the $\mathrm{H}_{\alpha}$ line from season to season. In the $\mathrm{Na}$ I $\mathrm{D}$ profile the signatures of accretion are almost absent, whereas the wind signatures are clearly expressed in the form of deep absorption at a radial velocity of about $-50 \mathrm{~km} / \mathrm{s}$. The depth of this absorption is an indicator of the wind density in the line-of-sight. Over the period between September and November 2016 this absorption totally disappeared and was absent until the end of observational season (February 2017). Figure 9 shows several superposed spectra of SU Aur in the region of D2 Na I. The broad profile belongs to photospheric absorption broadened by stellar rotation, $\mathrm{v} \sin i=60 \mathrm{~km} / \mathrm{s}$. Narrower absorption of variable intensity formed in the wind at a radial velocity of about $-50 \mathrm{~km} / \mathrm{s}$ is absent in the spectra obtained after November 20, 2016. At the same time, similar absorption in the $\mathrm{H}_{\alpha}$ line significantly weakened. At the beginning of the next season the wind signatures were seen again.

Such a fast disappearance of wind signatures points to a short scale of distances. Variations seemed to occur not in the disk wind that starts from the extended disk surface. There is likely a termination of outflowing on the magnetospheric boundary - propeller wind (see below), which could be caused by both an increase of the accretion rate and a decrease of the stellar magnetic field.

The interpretation of the observed variations in accretion and wind of CTTS became possible after developing methods of numerical modeling of magnetohydrodynamic processes (see the review of Romanova and Owocki, 2015). The basic parameters defining the accretion and wind modes: the stellar mass $M$, the stellar rotation angular velocity $\Omega$, the stellar magnetic field (magnetic dipole momentum $\mu$ ), and the mass accretion rate $\mathrm{M}_{\odot}$. Hence, one can determine the corotation radius $R_{\mathrm{co}}{ }^{3}=\mathrm{GM} / \Omega^{2}$ and the magnetosphere radius $\mathrm{R}_{\mathrm{m}}=\mu^{4} /\left(\mathrm{M}^{2} \cdot \mathrm{GM}_{*}\right)^{1 / 7}$. The condition $\mathrm{R}_{\mathrm{co}}=\mathrm{R}_{\mathrm{m}}$ determined the boundary between the accretion and propeller modes (Romanova and Owocki, 2015).

Magnetospheric accretion $\left(\mathrm{R}_{\mathrm{m}}<\mathrm{R}_{\mathrm{co}}\right)$ is substanitially unstable: the accretion disk "twists" the magnetosphere, there cyclically occurs an opening and reconnection of lines of 


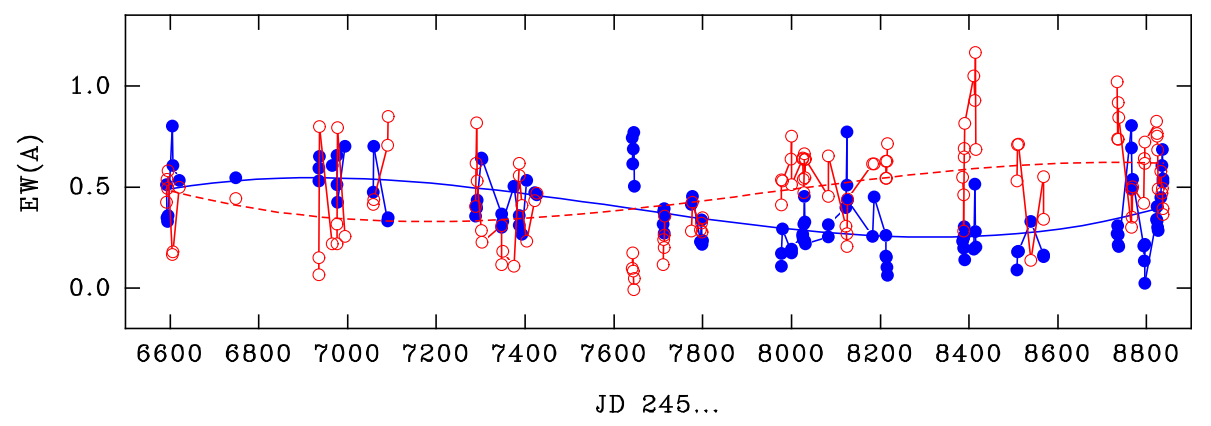

Fig. 8. Long-term variations of accretion and wind of RY Tau: variations of the equivalent width of Na I D absorptions formed in the flows of accretion (empty circles) and wind (filled circles) throughout seven years. The equivalent width of absorptions varied in the ranges of radial velocities of $-160 \ldots-30 \mathrm{~km} / \mathrm{s}$ (wind) and $+10 \ldots+150 \mathrm{~km} / \mathrm{s}$ (accretion). The solid and dotted curves are approximations by the third degree polinomial

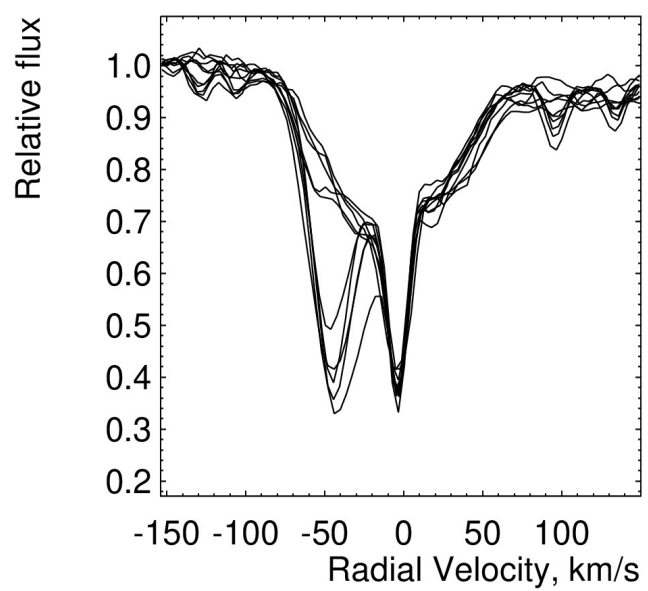

Fig. 9. Variations of the Na I 2 line profile in the spectrum of SU Aur (September - November, 2016). The radial velocity scale is astrocentric. The absorption peak near the zero velocity is interstellar absorption. The variable absorption detail at a radial velocity of about $-50 \mathrm{~km} / \mathrm{s}$ disappeared after November 20, 2016

force accompanied by "magnetospheric ejections" (Goodson et al., 1997; Zanni and Ferreira, 2013). In the propeller mode $\left(\mathrm{R}_{\mathrm{m}}>\mathrm{R}_{\mathrm{co}}\right)$ the centrifugal barrier prevents accretion, there occurs an outflow of matter, the "propeller wind" accelerated by magnetic centrifuge of the rotating magnetosphere. However, a detailed study of the propeller mode showed that episodic accretion is possible even in the strong propeller mode (Romanova et al., 2018). During short intervals the disk can approach the star, therefore accretion becomes possible on the magnetosphere boundary higher or lower than the disk plane. The characteristic recurrence time of such accretion episodes is tens of days. Thus, the outflow and accretion in the propeller mode are also unstable.

The RY Tau and SU Aur stars are related to rapidly rotating CTTS ( $\mathrm{v} \sin i \approx 50$ and $60 \mathrm{~km} / \mathrm{s}$, respectively) and therefore can be in the propeller mode, especially since RY Tau has a well-seen jet. In the propeller mode, the relative amount of matter ejected into the wind and its velocity increase with increasing ratio $\Omega_{*} / \Omega_{K}\left(\mathrm{R}_{\mathrm{m}}\right)$, where $\Omega_{*}$ is the stellar rotation angular velocity and $\Omega_{K}\left(\mathrm{R}_{\mathrm{m}}\right)$ is the angular velocity of the Keplerian disk rotation on the magnetosphere boundary
(Romanova et al., 2018). This means that an enhancement of the magnetic dipole $\mu$ (an increase of the magnetosphere radius $R_{m}$ ) can be observed by an observer as an increase in the velocity of the wind and radiation flux in the $\mathrm{H}_{\alpha}$ line.

The RY Tau and SU Aur stars are on the radiative evolutionary track, i.e., they have a radiative core and a convective envelope. In the case of detecting cyclic variations in the dynamics of accretion and wind with a characteristic time of a few years (see Fig. 8) one can suggest that the reason of such variations is magnetic activity of the young star. Moreover, cyclic variations in the rate of matter accretion onto the young star can be caused by the presence of a planet in the proximity to the magnetosphere (Teyssandier and Lai, 2020), as it is observed for CTTS CI Tau (Johns-Krull et al., 2016).

\section{Conclusions}

Accretion, wind, and dust are the three factors defining the observed activity of young stars. The basic source of activity is the accretion. The disk accretion supplies matter of the accretion disk onto the stellar magnetosphere boundary. The dusty disk wind is responsible for circumstellar extinction and irregular variability of CTTS. The stellar magnetosphere controls the matter transfer from the inner boundary of the accretion disk onto the stellar surface, as well as an outflow of part of the matter into the wind. Variations of the stellar magnetic field have effect on the dynamics of accretion and wind. Monitoring of accretion and wind can reveal the hidden processes - the cycle of magnetic activity of the young star or the planet's orbital motion.

Acknowledgements. The spectral observations of RY Tau and SU Aur with the ZTSh telescope in 2019-2021 including the processing and analysis of the derived spectra were supported by the grant of the Russian Science Foundation 19-72-10063. The author is grateful to S.A. Artemenko, E.V. Babina, K.N. Grankin, and S.Yu. Gorda for productive collaboration.

\section{References}

Akeson R.L., Walker C.H., Wood K., et al., 2005. Astrophys. J., vol. 622, p. 440. 
Alencar S.H.P. and Basri G., 2000. Astron. J., vol. 119, p. 1881.

Alencar S.H.P., Basri G., Hartmann L., Calvet N., 2005. Astron. Astrophys., vol. 440, p. 595.

Alencar S.H.P., Johns-Krull C.M., Basri G., 2001. Astron. J., vol. 122 , p. 3335.

Ambartsumyan V.A., 1949. Astron. zhurn., vol. 26, p. 3. (In Russ.)

Antipin S., Belinski A., Cherepashchuk A., et al., 2015. Inform. Bull. Var. Stars, vol. 6126, p. 1.

Babina E.V., Artemenko S.A., Petrov P.P., 2016. Astron. Lett., vol. 42(3), p. 193.

Bans A. and Koenigl A., 2012. Astrophys. J., vol. 758, p. 100.

Bouvier J., Alencar S.H.P., Harries T.J., et al., 2007. Protostars and Planets V, pp. 479-494.

Camenzind M., 1990. Rev. Modern Astron., vol. 3, p. 234.

Cranmer S.R., 2008. Astrophys. J., vol. 689, no. 1, pp. 316334.

Davies C.L., Kraus S., Harries T.J., et al. 2020. Astrophys. J., vol. 897, p. 31.

Dodin A., Grankin K., Lamzin S., et al., 2019. Mon. Not. Roy. Astron. Soc., vol. 482, p. 5524.

Dodin A.V., Lamzin S.A., 2012. Pis'ma v Astron. zhurn., vol. 38, no. 10, p. 1. (In Russ.)

Dodin A.V., Lamzin S.A., Chuntonov G.A., 2012. Astron. Lett., vol. 38, p. 167.

Dodin A., Lamzin S., Petrov P., et al., 2020. Mon. Not. Roy. Astron. Soc., vol. 497, p. 4322.

Dong R., Najita J.R., Brittain S., 2018. Astrophys. J., vol. 862 , p. 103

Dmitriev D.V., Grinin V.P., Katysheva N.A., 2019. Astron. Lett., vol. 45, no. 6, p. 371.

Folha D.F.M., Emerson J.P., 2001. Astron. Astrophys., vol. 365, p. 90.

Gahm G.F., Petrov P.P., Tambovsteva L.V., et al., 2018. Astron. Astrophys., vol. 614, p. 117.

Ghosh P., Lamb F.K., 1979. Astrophys. J., vol. 234, p. 296.

Giampapa M.S., Basri G.S., Johns C.M., Imhoff C., 1993. Astrophys. J. Suppl. Ser., vol. 89, p. 321.

Goodson A.P., Winglee R.M., Böhm, K.-H., 1997. Astrophys. J., vol. 489, p. 199.

Grinin V.P., Mitskevich A.S., Tambovtseva L.V., 2006. Astron. Lett., vol. 32, p. 110.

Grinin V.P., Arkharov A.A., Barsunova O.Yu., Sergeev S.G., Tambovtseva L.V., 2009. Astron. Lett., vol. 35, p. 114.

Gullbring E., Calvet N., Muzerolle J., Hartmann L., 2000. Astrophys. J., vol. 544, p. 927.

Günther H.M., Birnstiel T., Huenemoerder D.P., et al., 2018. Astron. J., vol. 156, p. 56.

Hartmann L., 1998. Accretion processes in star formation. New York : Cambridge University Press.

Hartmann L., Herczeg G., Calvet N., 2016. Ann. Rev. Astron. Astrophys., vol. 54, p. 135.

Herbig G.H., Bell K.R., 1988. Third Catalog of EmissionLine Stars of the Orion Population. Lick Observatory Bulletin No. 1111, Santa Cruz: Lick Observatory, Ca.

Johns C. and Basri G., 1995. Astron. J., vol. 109, p. 2800.

Johns-Krull C.M., McLane J.N., et al., 2016. Astrophys. J., vol. 26, p. 206.
Joy A.H., 1945. Astrophys. J., vol. 102, p. 168.

Koenigl A., 1991. Astrophys. J., vol. 370, p. L39.

Kurosawa R., Harries T.J., Symington N.H., 2006. Mon. Not. Roy. Astron. Soc., vol. 370, p. 580.

Kurosawa R., Romanova M.M., Harries T.J., 2011 Mon. Not. Roy. Astron. Soc., vol. 416, p. 2623.

Kwan J. and Fischer W., 2011. Mon. Not. Roy. Astron. Soc., vol. 411, p. 2383.

Labdon A., Kraus S., Davies C.L., et al., 2019. Astron. Astrophys., vol. 627, p. 36.

Lamzin S.A., Bisnovatyi-Kogan G.S., Errico L., et al., 1996. Astron. Astrophys., vol. 306, p. 877.

Long F., Herczeg G.J., Harsono D., et al., 2019. Astrophys. J., vol. 882, p. 49L.

Matt S. and Pudritz R.E., 2005. Astrophys. J., vol. 632, p. 135.

Petrov P.P., 2003. Astrophysics, vol. 46, p. 506.

Petrov P.P., Gahm G.F., Gameiro J.F., et al., 2001. Astron. Astrophys., vol. 369, p. 993.

Petrov P.P., Gahm G.F., Herczeg G.J., et al., 2014b. Astron. Astrophys., vol. 568, p. L10.

Petrov P.P., Gahm G.F., Stempels H.C., et al., 2011. Astron. Astrophys., vol. 535, p. 6.

Petrov P.P., Grankin K.N., Gameiro J.F., et al., 2019. Mon. Not. Roy. Astron. Soc., vol. 483, p. 132.

Petrov P.P., Gullbring E., Ilyin I., et al., 1996. Astron. Astrophys., vol. 314 , p. 821.

Petrov P.P., Kurosawa R., Romanova M.M., et al., 2014a. Mon. Not. Roy. Astron. Soc., vol. 442, p. 3643.

Pevtsov A.A., Fisher G.H., Acton L.W., et al., 2003. Astrophys. J., vol. 598, p. 1387.

Rei A.C.S., Petrov P.P., Gameiro J.F., 2018. Astron. Astrophys., vol. 610, p. A40.

Reipurt B., Pedrosa A., Lago M.T.V.T., 1996. Astron. Astrophys. Special Suppl. Ser., vol. 120, p. 229.

Rodriguez J.E., Loomis R., Cabrit S.E, et al., 2018. Astrophys. J., vol. 859, p. 150.

Romanova M.M. and Owocki S.P., 2015. Space Sci. Rev., vol. 191, p. 339.

Romanova M.M., Blinova A.A., Ustyugova G.V., et al., 2018. New Astronomy, vol. 62, pp. 94-114.

Romanova M.M., Ustyugova G.V., Koldoba A.V., Lovelace R.V.E., 2009. Mon. Not. Roy. Astron. Soc., vol. 399, p. 1802.

Shenavrin V.I., Petrov P.P., Grankin K.N., 2015. Inform. Bull. Var. Stars, vol. 6143, p. 1.

Shu F., Najita J., Ostriker E., et al., 1994. Astrophys. J., vol. 429, p. 781.

Takami M., Wei Y.-J., Chou M.-Y., et al., 2016. Astrophys. J., vol. 820, p. 139.

Tambovtseva L.V. and Grinin V.P., 2008. Astron. Lett., vol. 34 , p. 231

Teyssandier J. and Lai D., 2020. Mon. Not. Roy. Astron. Soc., vol. 495 , p. 3920.

Valenti J.A. and Piskunov N., 1996. Astron. Astrophys. Suppl. Ser., vol. 118, p. 595.

White R.J. and Ghez A.M., 2001. Astrophys. J., vol. 556, p. 265.

Zanni C. and Ferreira J., 2013. Astron. Astrophys., vol. 550, p. A99. 